\title{
INCIDENCIA DE DISFAGIA EN PACIENTES CON ENFERMEDAD RESPIRATORIA CRÓNICA QUE ASISTEN A UN PROGRAMA DE REHABILITACIÓN PULMONAR DEL NORORIENTE COLOMBIANO
}

\author{
Leslie Vargas-Ramírez ${ }^{1}$, Diana Cano ${ }^{1}$, Fabio Bolívar ${ }^{1}$, Lizeth Rodríguez ${ }^{1}$, Katherine Rincón ${ }^{1}$, Juliana Suarez ${ }^{1}$ \\ Laura García ${ }^{2}$, Ana Jiménez² \\ 1. Instituto Neumológico del Oriente, 2. Universidad Industrial de Santander. Bucaramanga, Colombia.
}

INTRODUCCIÓN: La presencia de disfagia puede traer consecuencias graves incluyendo aspiraciones o episodios de asfixia. En los pacientes con enfermedad respiratoria crónica ERC constituye una de las causas de mal control de la enfermedad.

OBJETIVO: Describir la incidencia de disfagia en pacientes con ERC que asisten a un programa de rehabilitación pulmonar-RP del nororiente colombiano

METODOS: Estudio analítico de cohorte retrospectiva en pacientes mayores de 18 años que ingresaron a un programa de RP y finalizaron las 48 sesiones, con datos iniciales y finales de la escala EAT-10(Eating Assessment Tool-10), útil en el cribado de disfagia. Se muestran datos de frecuencias relativas y absolutas. La incidencia se calculó con la población a riesgo al inicio del programa ( $n: 32)$.

RESULTADOS: Se analizaron datos de 38 pacientes. La mediana de edad 70 años, 55,3\% mujeres, diagnostico principal fue EPOC (52,6\%). El 84,2\% presento al inicio un EAT-10, negativo. El 33,3\% de pacientes con EAT10-ES positivos (puntaje>3) tenían como diagnóstico asma (tabla1). La incidencia de disfagia fue del 12,5\% (tabla 2), el diagnóstico con mayor casos incidentes fue EPOC (75\%) (tabla 3).

Tabla 1. Test EAT-10 de acuerdo al diagnóstico en la población de estudio

\begin{tabular}{lccc}
\multicolumn{4}{c}{ Test EAT-10 } \\
\multicolumn{1}{c}{ Diagnóstico } & Negativo & Positivo & Total \\
& $n(\%)$ & $n(\%)$ & \\
\hline EPOC & $19(59,4)$ & $1(16,7)$ & $20(52,6)$ \\
Asma & $3(9,4)$ & $2(33,3)$ & $5(13,2)$ \\
Fibrosis pulmonar & $2(6,3)$ & $1(16,8)$ & $3(7,9)$ \\
Disnea & $2(6,3)$ & $1(16,7)$ & $3(7,9)$ \\
EPID & $3(9,4)$ & $0(0,0)$ & $3(7,9)$ \\
Otros & $3(9,4)$ & $1(16,7)$ & $4(10,5)$ \\
\hline Total & $32(100,0)$ & $\mathbf{6 ( 1 0 0 , 0 )}$ & $\mathbf{3 8 ( 1 0 0 , 0 )}$ \\
\hline
\end{tabular}

Tabla 2. Cambio en la clasificación del EAT-10 posterior a las cuatro fases del programa de rehabilitación pulmonar.

\begin{tabular}{|c|c|c|c|}
\hline \multirow[b]{2}{*}{ EAT-10 Inicial } & \multicolumn{2}{|c|}{ EAT-10 final } & \multirow[b]{2}{*}{$\begin{array}{l}\text { Total } \\
\text { n (\%) }\end{array}$} \\
\hline & $\begin{array}{c}\text { Negativo } \\
\text { n (\%) }\end{array}$ & $\begin{array}{c}\text { Positivo } \\
\text { n (\%) }\end{array}$ & \\
\hline Negativo & $28(87,5)$ & $4(12,5)$ & $32(100.0)$ \\
\hline Positivo & $5(83,3)$ & $1(16,7)$ & $6(100,0)$ \\
\hline Total & $33(86,8)$ & $5(13,2)$ & $38(100,0)$ \\
\hline
\end{tabular}

Tabla 3. Incidencia de disfagia en la población de estudio, general y por diagnóstico

\begin{tabular}{lcc} 
Variables & $\begin{array}{c}\text { No incidente } \\
\mathbf{n}(\%)\end{array}$ & $\begin{array}{c}\text { Incidente* } \\
\mathrm{n}(\%)\end{array}$ \\
\hline $\begin{array}{l}\text { Disfagia } \\
\text { Diagnóstico }\end{array}$ & $28(87,5)$ & $4(12,5)$ \\
EPOC & $16(57,1)$ & $3(75,0)$ \\
Asma & $3(10,7)$ & $0(0,0)$ \\
Fibrosis pulmonar & $2(7,1)$ & $0(0,0)$ \\
Disnea & $1(3,6)$ & $1(25,0)$ \\
EPID & $3(10,7)$ & $0(0,0)$ \\
Otros & $3(10,7)$ & $0(0,0)$ \\
\hline
\end{tabular}

CONCLUSIÓN: La disfagia es un trastorno frecuentemente no diagnosticado a pesar de su alta prevalencia y de su impacto clínico. Conocer los datos de incidencia de la disfagia nos permitirá establecer intervenciones en los pacientes del programa de RP a fin de disminuir las consecuencias en el control de la enfermedad respiratoria. 